\title{
Éducation en Thaïlande : la croissance économique ne suffit plus
}

Education in Thailand: economic growth is not enough

Educación en Tailandia : el crecimiento económico no basta

\section{Sandrine Michel}

\section{(2) OpenEdition}

Journals

Édition électronique

URL : http://journals.openedition.org/ries/3691

DOI : 10.4000/ries.3691

ISSN : 2261-4265

Éditeur

Centre international d'études pédagogiques

Édition imprimée

Date de publication : 15 avril 2014

Pagination : 89-98

ISBN : 978-2-85420-603-6

ISSN : $1254-4590$

\section{Référence électronique}

Sandrine Michel, «Éducation en Thaïlande : la croissance économique ne suffit plus », Revue

internationale d'éducation de Sèvres [En ligne], 65 | avril 2014, mis en ligne le 15 avril 2016, consulté le 06 janvier 2020. URL : http://journals.openedition.org/ries/3691 ; DOI : 10.4000/ries.3691

\section{(c) Tous droits réservés}




\section{Éducation en Thaïlande : \\ la croissance \\ économique ne suffit plus}

\section{Sandrine Michel}

Aujourd'hui, le mode de croissance des pays émergents du Sud-Est asiatique est bien connu (World Bank, 1993). À partir du milieu des années 1960, dans un contexte d'augmentation des revenus des ménages, un mode de consommation restrictif a permis de dégager une forte épargne intérieure. Dans le même temps, une intervention publique incitative, encadrant des secteurs financiers nationaux balbutiants, a autorisé un taux d'investissement interne élevé dans les industries de biens de consommation utilisatrices d'une main d'œuvre abondante et peu chère. Ces pays ont accédé aux transferts technologiques au prix fort, en important des biens d'équipement. Leur insertion dans la division internationale du travail a donc reposé sur un régime de croissance tiré par les exportations et sur des marchés intérieurs en expansion. À long terme, cette croissance extensive n'est soutenable que dans la mesure où elle suscite et entretient une croissance de la productivité et en assure une certaine redistribution sous forme d'augmentation de revenus. Dans ces conditions, dans la plupart de ces pays, le retour de la croissance économique après la crise financière de 1997 (Stiglitz, 2002) a posé à nouveau la question de son partage (Witte, 2000 ; Khoman, 2005). Mais un questionnement sur ses conditions structurelles, plus inédit, a également émergé.

En effet, avec la croissance économique, les pays émergents ont connu une rupture dans le rythme de développement de leurs dépenses publiques et sociales. Ainsi, les travaux fondateurs de l'étude de la contribution de l'éducation au processus de croissance asiatique ont montré le développement extrêmement rapide des systèmes éducatifs nationaux et le rôle des dépenses publiques (Tan \& Mingat, 1989). Ils ont contribué à forger l'idée de la performance scolaire des pays asiatiques (Psacharopoulos, 1991). Cela étant, l'action conjointe de facteurs structurels, tels que le niveau et le rythme de développement des dépenses publiques d'éducation, la mobilité sociale associée à l'éducation ou encore le rendement de l'investissement en éducation, privé comme public, ont bien été mis en avant sans, toutefois, avoir été analysés comme des vecteurs coordonnés d'un régime de croissance. 
Du point de vue quantitatif, les performances éducatives de ces pays sont indéniables (Watson, 1982 ; Sinnathambu, 2003). Toutefois, la dynamique de leur développement éducatif présente des limites liées à la fois à leurs types de spécialisation internationale et à l'insertion d'une éducation, désormais généralisée, dans leurs structures sociales. Cet article cherche, à partir du cas de la Thaïlande, à mettre en évidence la manière dont le régime de croissance asiatique a généré un modèle de développement de l'éducation et à s'interroger sur le caractère soutenable de ce dernier à long terme ${ }^{1}$.

\section{LES ÂGES DE LA CROISSANCE ÉDUCATIVE EN THAÏLANDE}

L'analyse quantitative de long terme (1900-2011) permet d'identifier les principales phases de développement du système éducatif thaïlandais.

Graphique 1. Effectifs scolaires totaux de 1911 à 2011

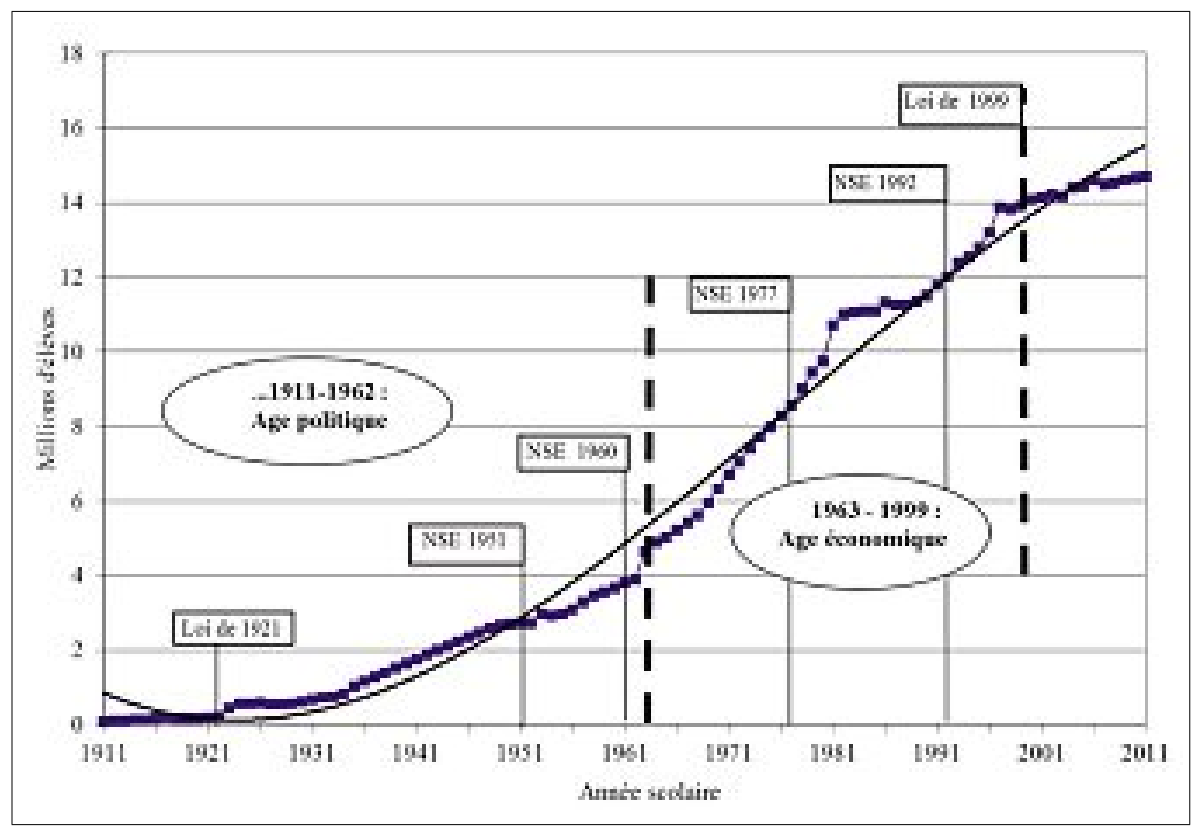

Sources : Auteur - Base de données de Citizenship Education \& Longitudinal Survey (CELS)

1. Cet article est issu d'un travail réalisé au sein du Center for Education and Labour Studies de l'Université de Chaing Mai en Thaïlande pour l'Institut de recherche pour le développement (IRD), consultable dans Michel S. (2010) “The Burgeoning of Education in Thailand : a quantitative success" : 11-38, in : Mounier A., Tangchaung P., (2010) Education and Knowledge in Thailand, Bangkok : Silkworm Books. 


\section{L’âge politique : éducation et modernisation de la nation (1900-fin des années 1950)}

L'histoire éducative de la Thaillande (Ministry of Education, 1976) s'ouvre à la fin du XIX ${ }^{\mathrm{e}}$ siècle. Jusque dans les années 1960, elle obéit à un déterminant politique. La modernisation de la nation est la réponse, formulée par les groupes sociaux au pouvoir, notamment l'aristocratie dirigeante et cosmopolite, à la menace coloniale représentée par le Royaume-Uni et la France. Le rapport social dominant, fondé sur les appartenances locales et communautaires, faisait obstacle à ce projet de société. L'éducation sera le vecteur indiscutable par lequel l'État développera un rapport social de substitution, fondé sur une relation directe et individuelle entre le roi et ses sujets au détriment des anciennes références. Dans ces conditions, l'éducation constitue un vecteur fondamental de la modernisation de la nation.

Par une politique publique méticuleuse et volontariste, l'éducation devient alors un élément d'homogénéité et d'identification nationales, fondé sur des structures sociales de type individuel, indispensable à l'essor d'une économie de marché (Wyatt, 1969). Elle concerne essentiellement l'enseignement primaire mais aussi le supérieur. La première université, Chulalongkorn University, fut ainsi créée en 1917. Sa mission était de former la haute administration royale.

\section{L’âge économique : l'éducation, un soutien de la croissance (1960-1999)}

La seconde période s'ouvre au début des années 1960 et s'achève en 1999. Il s'agit d'une phase de croissance très rapide du système éducatif qui passe, en quarante ans, de 4 à 14 millions d'élèves. Elle répond davantage à un déterminant économique (Navaneetham, 2002). L'éducation soutient alors le processus de croissance économique, en favorisant la croissance relative de la productivité du travail. Ce faisant, elle assure le maintien dynamique des avantages comparatifs thaïs sur les marchés internationaux.

Dans le développement éducatif contemporain (1960-1999), trois facteurs structurels agissent selon des configurations historiques originales.

\section{La démographie}

Dans ce pays, le processus de croissance économique a coïncidé avec la transition démographique. L'essor de la scolarisation y a bénéficié de deux influences démographiques très favorables.

La première concerne les effectifs scolarisés. L'effondrement du taux de fécondité a pour conséquence, à la fin des années 1970, la décroissance du nombre d'élèves à scolariser en même temps que s'élève, pour un nombre croissant, leur carrière scolaire, d'abord lentement à travers le primaire puis, de plus en plus rapidement, à travers le secondaire et le supérieur. 
La seconde influence concerne la population supportant l'effort de scolarisation. Au moment de l'essor du système éducatif, les enfants des cohortes de la forte croissance démographique passée sont devenus des adultes actifs, sur lesquels repose le financement de l'effort d'éducation. Par conséquent, les progrès de la scolarisation de générations toujours moins denses sont répartis durant une vingtaine d'années entre des adultes plus nombreux. Mais le ratio qui rapporte le nombre d'élèves aux actifs a cessé de baisser depuis le début des années 1990, soulignant que tout effort financier supplémentaire en faveur de l'éducation sera désormais plus difficile.

Au total, les progrès de la scolarisation ont donc été réalisés au cours d'une conjoncture démographique exceptionnelle. Mais cette dernière a finalement constitué un cadre passif dont les opportunités n'ont pas été activées (Janjoren, 1985). Son retournement pose la question de la soutenabilité du développement scolaire actuel, comme le pointe déjà le ralentissement de la croissance de quelques indicateurs, tels que les effectifs du secondaire par exemple.

\section{L'aversion à l'inégalité}

À partir de données longitudinales, les carrières scolaires des cohortes, définies par l'année d'entrée en primaire, mettent en évidence le caractère transitoire des inégalités d'accès à l'éducation.

\section{Graphique 2. Taux de réalisation par niveau d'éducation et par génération complète d'entrants en primaire}

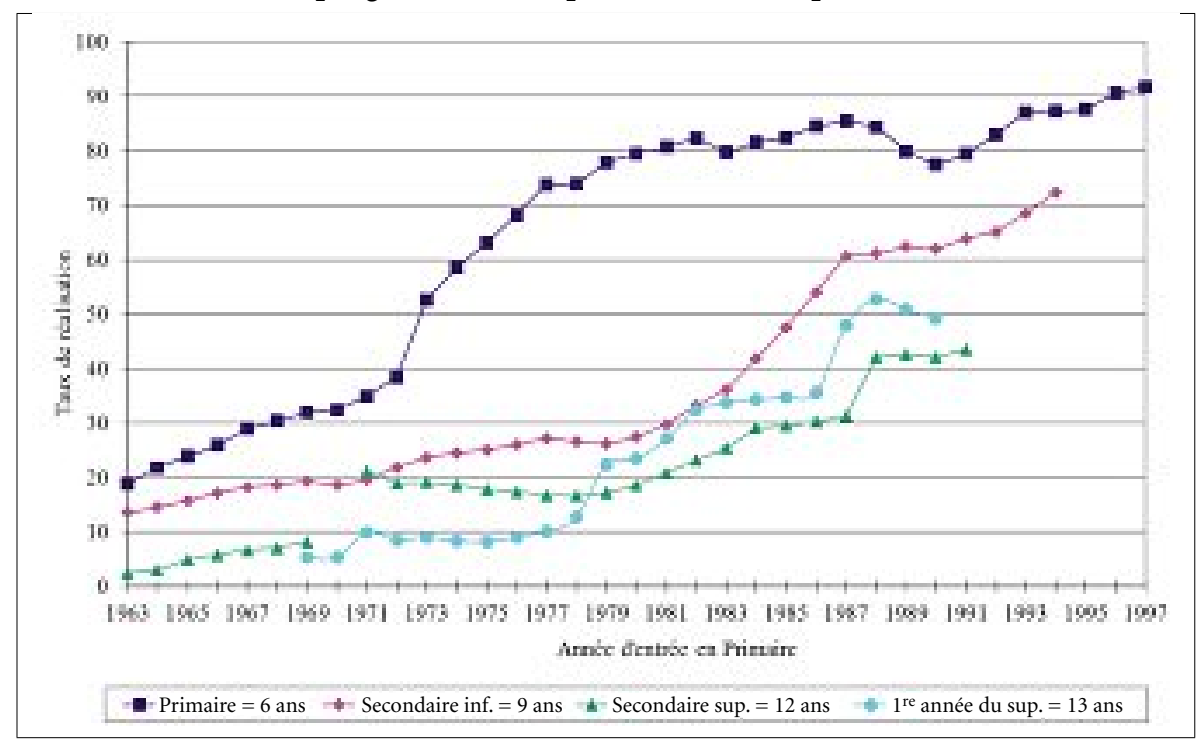

Source : Auteur - Base de données CELS

Lecture du graphique : Parmi les enfants qui entrent en primaire en 1963, $19 \%$ suivront un cycle primaire complet, $12 \%$ finiront leur $1^{\text {er }}$ cycle de secondaire et seulement $2,5 \%$ accèderont au secondaire supérieur. 
À l'ouverture maximale des destins scolaires pour peu d'élèves (pour les cohortes de 1963 à 1972) succède une période au cours de laquelle un grand nombre d'élèves (cohortes de 1973 à 1980) accèdent à un destin scolaire réduit puisque limité au primaire. L'inégalité des destins scolaires est alors maximale, puisque c'est toujours la même proportion d'une génération qui accède au secondaire alors que celle qui achève son primaire explose littéralement.

À partir des cohortes des années 1980, les inégalités de destin scolaire commencent à se refermer. Il est assez intéressant de noter que cette tendance est basée sur une progression simultanée de la réalisation des deux cycles secondaires et de l'accès au supérieur et non sur des réalisations successives de cycle en cycle. Pour les dernières générations complètes disponibles, cette dynamique égalitaire est moins active. Aujourd'hui, les taux de réalisation élevés du secondaire rendent plus difficiles tout nouveau progrès, renvoyant sans doute à la question du coût de l'égalité.

\section{L'évolution de la dépense publique d'éducation}

Replacée dans le contexte plus large de la croissance économique, la dépense publique d'éducation est de nature pro-cyclique, dans le sens où ses fluctuations suivent celles de la richesse nationale. La dépense publique d'éducation, tout comme le budget de l'État, est donc liée à la prospérité économique. Une croissance économique soutenue va favoriser les progrès de l'éducation via un accroissement proportionnel des prélèvements publics; des difficultés vont, au contraire, la ralentir.

\section{Depuis 1999 : une transition, pour aller où ?}

La loi éducative de 1999 modifie profondément le système éducatif thaï. Elle le fait tout d'abord en généralisant l'insertion du premier cycle secondaire dans l'enseignement obligatoire, qui passe ainsi de 6 à 9 ans. Mais cette loi ouvre également des débats plus fondamentaux.

Le premier concerne la possibilité de substituer des financements privés, essentiellement des ménages, aux financements publics. Il pose donc la question du périmètre de l'éducation publique en fonction des financements. Cette position est justifiée par le fait que la croissance économique continue de se traduire par un accroissement du revenu moyen. Le second concerne l'individualisation du rapport au savoir, et sa nécessaire dimension critique, dans une société hiérarchique. 


\section{UN BILAN DU MODÈLE \\ DE DÉVELOPPEMENT DE L’ÉDUCATION EN THAïlLANDE}

Le bilan du modèle de développement de l'éducation en Thaïlande est considérable (Collins \& Rhoads, 2008). Les taux de scolarisation, global ou par niveau, ont bondi dans des temps très courts. Toutes choses égales par ailleurs, l'image selon laquelle la Thaïlande accomplit en matière de scolarisation en une quarantaine d'années ce que la France a réalisé sur près d'un siècle et demi peut être mobilisée. Rappelons que le système éducatif thaïlandais a permis aux générations nombreuses d'enfants des années 1970 de poursuivre des scolarisations de plus en plus longues pour le plus grand nombre, dans un temps très court. Rappelons aussi que cette massification du système éducatif a bénéficié de circonstances démographiques exceptionnelles.

Mais, plus fondamentalement, la Thaïlande a financé sa croissance éducative par sa croissance économique. C'est le rythme de cette dernière qui a fixé celui des financements publics d'éducation via l'évolution pro-cyclique du budget de l'État. La conjoncture démographique a favorisé cet effort proportionnel, en dégageant des marges que la seule croissance économique n'aurait pas permises. En effet, des années 1960 jusqu'au milieu des années 1980, l'effort financier de la nation en faveur de l'éducation évolue modérément dans un contexte d'accroissement rapide des effectifs. Lorsque, à la fin des années 1970, la transition du primaire, en cours d'achèvement, vers le secondaire se pose, cet effort ne suffit plus. Les besoins croissants du secondaire (ONEC, 2001) sont couverts temporairement par un transfert des moyens du primaire, dont les effectifs commencent à décroître du fait de l'évolution démographique. Cette solution conjoncturelle s'épuise rapidement. Il en va de même de l'utilisation de la faible inflation par l'État que génère la croissance thaïe.

À côté de ces éléments d'adaptation opportunistes, dont l'histoire éducative de la Thaïlande fourmille, la dépense publique par niveau entame une progression inédite au début des années 1980. Tous les niveaux du système éducatif en bénéficient. Cette croissance cache cependant des disparités. Ainsi la constance de l'effort pour le primaire fera de ce niveau un socle robuste du système éducatif (World Bank, 1998).

La scolarisation dans le secondaire s'accélère considérablement à la fin des années 1980 jusqu'à la crise de 1997 (Tunsiri, 1994 ; World Bank, 2001) sans que la dépense par élève n'accompagne véritablement cet essor. Au cours de cette période, la scolarisation dans le secondaire se poursuit dans le secondaire supérieur, atténuant considérablement le verrou que constitue l'organisation bicéphale de ce niveau du système éducatif (Jones, 2003). La crise économique et financière de 1997 mettra un terme à la croissance de la dépense par élève, même si la politique publique évite tout rationnement du niveau primaire et tente de préserver le secondaire. 
La croissance de la dépense par élève dans le supérieur devance son essor démographique. Ici, deux éléments retiennent l'attention. D'une part, l'enseignement supérieur s'est massifié brusquement au début des années 1970, en réponse à la contestation politique de la jeunesse thaïe selon un principe qui a préservé une profonde sélectivité sociale: l'accès aux open universities est libre et peu onéreux mais les contreparties en termes de qualité académique ou de reconnaissance des titres sont nulles. La normalisation des open universities débute à partir de 1982. D'autre part, l'enseignement supérieur, mieux organisé, se diversifie : l'énorme besoin de production d'enseignants s'estompe au profit d'autres spécialités mais très lentement. Les poursuites d'études à l'étranger demeurent importantes pour les couches les plus aisées de la population. Cependant, avec 2 millions d'étudiants, l'enseignement supérieur national doit désormais faire face aux poursuites classiques d'études en provenance du secondaire, avec des structures normées par d'autres déterminants que celui de la massification: élitisme, faiblesse numérique et spécialisation dans la production de masse d'enseignants.

Par ailleurs, la performance quantitative du système éducatif fait désormais place à une interrogation qualitative généralisée. Aujourd'hui, en dépit de l'inachèvement de la scolarisation secondaire et de la médiocrité des poursuites d'études dans le supérieur, la croissance éducative est interrompue. La question de la soutenabilité de ce mode de développement semble donc posée.

\section{DÉPENSE PUBLIQUE LIMITE? UNE LIMITE PEUT EN CACHER UNE AUTRE...}

La Thaillande reprend à son compte le vieux débat économique portant sur l'existence d'une dépense publique limite et l'applique à l'éducation. Au titre de ce concept, avancé par Peacock \& Wiseman en 1953 et régulièrement reformulé depuis, en particulier en phase de crise économique ou financière, il existerait une taille de l'État soutenable pour une conjoncture économique donnée. L'augmentation de la sphère publique au-delà de la limite ralentirait la croissance.

En Thaillande, le périmètre actuel de l'éducation publique est fixé autour des $4 \%$ du PIB ou des $25 \%$ du budget national. Ces niveaux de contribution semblent être interprétés comme autant de limites. C'est ce que suggère leur stabilité face à des besoins quantitatifs et qualitatifs d'éducation toujours croissants. Pour faire face à ces derniers, et compte tenu de l'existence supposée de cette dépense publique limite, d'autres solutions sont expérimentées. Parmi elles, les plus significatives sont de nature institutionnelle. Ainsi, en 2002, le secondaire supérieur et l'enseignement supérieur ont été exclus du périmètre de l'éducation publique. 
En optant pour ce type de choix, la politique publique a opposé aux limites du développement éducatif actuels deux dispositifs institutionnels en rupture avec les principes politiques qui ont, jusqu'ici, guidé la promotion de l'éducation en Thaïlande.

La première rupture introduite par la réforme de la politique publique d'éducation concerne le développement de l'éducation conçue comme un bien public, central dans la correction des inégalités sociales. En cela, l'éducation est un dispositif de la modernité, développé depuis la fin du XIX ${ }^{\mathrm{e}}$ siècle. L'accès, désormais privé, aux plus hauts niveaux de formation initiale porte donc sur le pacte fondateur de la nation, au moment même où l'éducation, en dépit de ses limites, s'affirme comme un moyen robuste d'accéder à de meilleures positions sociales.

La seconde rupture concerne le périmètre du système éducatif public. À structures économiques constantes, la question de la soutenabilité du rythme de croissance des effectifs scolarisés a été arbitrée par une restriction du périmètre du système public d'éducation du primaire jusqu'au secondaire inférieur et par un transfert vers les ménages du coût de l'accès aux plus hauts niveaux de formation initiale (secondaire supérieur et enseignement supérieur). Cette restriction du domaine public de l'éducation est sans aucun doute l'une des conséquences de la crise économique de 1997. Elle ne saurait pourtant s'y 96 confondre. En effet, compte tenu des besoins pour accroître la qualité du premier degré de l'enseignement secondaire, compte tenu des niveaux de scolarisation atteints pour le secondaire supérieur et pour l'enseignement supérieur, le maintien des taux de scolarisation et a fortiori leur développement supposent un accroissement substantiel des financements publics.

Pour l'instant, le financement du développement du secondaire supérieur et du supérieur lui-même repose donc sur les ménages, sur la base d'une anticipation de gains futurs élevés (Blaug, 1971). Ce calcul, déjà expérimenté par le système éducatif thaï pour le développement du secondaire professionnel, a échoué dans les années 1990, en raison du niveau de revenus des ménages concernés par ce niveau éducatif intermédiaire. Plus globalement, le calcul d'optimisation inter-temporelle de l'investissement privé en éducation (Pholphirul, 2005) se heurte au régime de bas salaire qui fonde la spécialisation internationale de la Thaïlande.

Comme nous l'avons évoqué, ces choix publics sont fortement tributaires de la notion de dépense publique limite. Mais, en Thaïlande comme ailleurs, cette notion renvoie à des conditions structurelles plus larges que le simple système éducatif lui-même. Sont en effet concernés la répartition de la richesse, la dynamique du marché du travail ou encore le caractère incomplet du rapport salarial (Boyer, 2002).

Les progrès de la scolarisation, désormais généralisée, se heurtent en effet au régime de basse qualification, fortement désincitatif pour l'élévation du 
niveau d'éducation. La faible différenciation salariale selon le niveau de formation initiale et le quasi plein-emploi se traduisent par une très forte mobilité de l'emploi (certaines entreprises industrielles enregistrent un turn-over annuel proche ou égal à $100 \%$ ), ainsi que par une très forte mobilité de statut entre travail salarié et travail indépendant (Michel \& Oudin, 2003). De plus, ce régime de basse qualification génère un effet de seuil, bridant la croissance de la productivité. Cette situation altère la position concurrentielle de la Thaillande sur les marchés internationaux, ainsi que le dynamisme de son marché intérieur. Le caractère durable de ces limites structurelles du régime de croissance fait émerger la question de la transition vers un nouveau régime de travail et du rôle que pourrait y jouer le système éducatif.

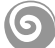

Ce travail préliminaire sur la Thaillande nous permet de constater que, par le biais de l'insertion internationale de cette économie, l'éducation est bien une composante structurelle de la croissance. Les limites auxquelles se heurte aujourd'hui le développement éducatif sont le fruit de la faible valorisation du travail éduqué et du traitement restrictif, en termes de revenus actuels ou actualisés, dont ce dernier fait l'objet. Sont ici en cause l'étroitesse des créneaux en termes de spécialisation internationale et la véritable limitation dont font l'objet les salaires. Dans le même temps, l'éducation, identifiée comme un soutien à la croissance, fait l'objet d'une politique publique durable désormais confrontée à la nécessité d'une révision de la contribution de tous les revenus à l'entretien de la dynamique économique.

\section{BIBLIOGRAPHIE}

BLAUG M. (1971) : The rate of return to investment in education in Thailand, A report to the National Education Council on the Third Educational Development Plan, 1971.

BOYER R. (2002) : "Perspectives on the Wage-Labour Nexus": 73-79, in: Boyer R., Saillard Y. (2002) Regulation Theory: The State of the Art [1995], London and New York: Routledge.

COLLINS C. \& RHOADS R. (2008) : "The World Bank and Higher Education in the Developing World: the Cases of Uganda and Thailand", International Perspectives on Education and Society, $9: 177-221$.

JANJAROEN W. S. (dir.) (1985): Economic Impact of Demographic Change on Education Planing in Thailand; 1987-2001, Thailand Development Research Institute Foundation, Bangkok: TDRI.

JONES G.W. (2003) : "Strategies and Achievements in Expanding Lower Secondary Enrollments: Thailand and Indonesia", Asian Metacentre Research Paper Series $n^{\circ} 13$, Asian MetaCentre for Population and Sustainable Development Analysis, Headquarters at Asia Research Instititute - National University of Singapore. 
KHOMAN S. (2005) : "Education - The Key to Long-term recovery?", in: Warr P. (2005) Thailand Beyond The Crisis, London and New York: Routledge Curzon: Research on Southeast Asia.

MICHEL S. \& OUDIN X. (dir.) (2003) : La mobilisation de la main-d'œuvre, Paris : L'Harmattan-IRD (Travail et Mondialisation).

Ministry of Education (1976) : A History of Thai Education, Bangkok: The Ministry.

NAVANEETHAM K. (2002): "Age Structural Transition and Economic Growth: Evidences from South and Southest Asia", Asian Metacentre Research Paper Series $\mathrm{n}^{\circ}$ 7, Asian MetaCentre for Population and Sustainable Development Analysis, Headquarters at Asia Research Institute - National University of Singapore.

Office of the National Education Commission (2001) : National Case Study on Policy Issues in Diversification of Secondary Education, Bangkok: ONEC.

PHOLPHIRUL P. (2005) : Competitiveness, Income Distribution, and Growth in Thailand: What Does the Long-run Evidences Show?, International Economic Relations Program, Bangkok: TDRI.

PSACHAROPOULOS G. (1991) : The Economic Impact of Education: lessons for policy makers, San Francisco, California: ICF Press.

SINNATHAMBU A. (2003) : "Education for All: Towards Equality and Accessibility in Thailand", Institute for Social and Economic Studies, Working Paper $n^{\circ} 2$.

TAN J.-P \& MINGAT A. (1989) : Educational Development in Asia: A Comparative Study Focussing on Cost and Financing Issues, International Discussion Paper - Asia Regional Studies, Report n ${ }^{\circ}$ IDP 51, The World Bank.

TUNSIRI V. (1994) : Secondary Education in Thailand, Bangkok: Office of National Education Commission.

STIGLITZ J. (2002) : La grande désillusion, Fayard: Paris (chapitre 4).

WATSON K. (1982) : Educational Development in Thailand, Hong Kong: Heinemann Asie.

PEACOCK A. T. \& WISEMAN J. (1953) : "The Growth of Public Expenditure in the United Kingdom", NBER.

World Bank (1993): The East Asian Miracle, Economic Growth and Public Policy, Washington: Oxford University Press.

World Bank (1998) : Thailand Education Achievements, Issues and Policies, Education Sector Unit, Report n ${ }^{\circ} 18417-\mathrm{TH}$.

World Bank (2001) : Secondary Education for Employment, volume 1: A Policy Note, Report n $22660-\mathrm{TH}$.

WITTE J. (2000) : Education in Thailand after the Crisis: a Balancing Act between Globalization and National Self-Contemplation", International Journal of Educational Development, $20: 223-245$.

WYATT D. (1969) : The Politics of Reform in Thailand: Education in the Reign of King Chulalongkorn, New Haven and London: Yale University. 